\title{
PAPERS
}

\section{On the total internal reflection frustrated by a dielectric slab: Goos-Hänchen shift and deformation of the beam-wave profile}

\author{
Jozefa Červeňová, Rastislav Dosoudil, \\ Jaroslav Franek, L’ubomír Šumichrast*
}

\begin{abstract}
The total internal reflection of a beam wave on planar dielectric boundary in presence of a nearby dielectric slab is thoroughly investigated together with its influence on the Goos-Hänchen shift and on the beam-wave-profile deformation.

K e y w or d s: electromagnetic wave, total reflection, Goos-Hänchen shift, beam-wave-profile
\end{abstract}

\section{Introduction}

Reflection and transmission of a homogeneous plane wave at the plane boundary of two dielectric media is a well known phenomenon commonly treated in nearly all standard textbooks. Investigation of the total internal reflection of inhomogeneous plane waves and spatially confined beam waves is subject of permanent interest since the discovery of the Goos-Hänchen shift [1]. There is a huge number of papers published over the last seventy years concerning this topic, just a small nonrepresentative sample is for illustration exemplified by [2$6]$.

In [7] we have investigated the effects occurring due to the total internal reflection on a simple boundary of an inhomogeneous plane wave with a sinusoidal profile - the classical Goos-Hänchen shift - and subsequently we have considered a spatially-confined periodic beam wave. The results lead to the conclusion that the maximum shift of the beam axis due to the total internal reflection is about one quarter of the beam width.

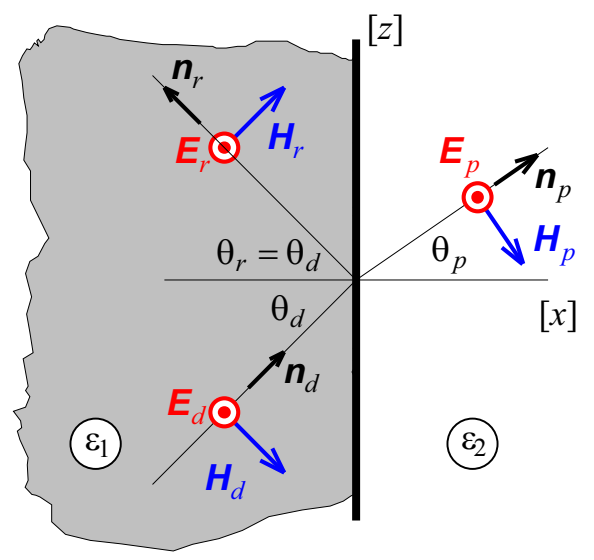

In this subsequent paper the proximity effects of a dielectric slab on the totally reflected beam wave at the planar dielectric interface are investigated. They are presented for both polarisations, for the TE-polarised-wave case, as well as for the TM-polarised-wave case.

2 Standard approach to the plane wave reflection and transmission on plane boundaries

Within the framework of the standard treatment of wave reflection and refraction on the planar boundary as depicted in Fig. 1, the electric intensity vectors of the incident, reflected and refracted homogeneous TEpolarised plane wave are expressed as [7]

$$
\begin{aligned}
& \boldsymbol{E}_{d}(\boldsymbol{r})=\boldsymbol{u}_{y} E_{d} \exp \left(-j \beta_{1} \boldsymbol{n}_{d} \cdot \boldsymbol{r}\right)= \\
& =\mathbf{u}_{y} E_{d} \exp \left(-j q_{1} x\right) \exp (-j k z), \\
& x<0,
\end{aligned}
$$

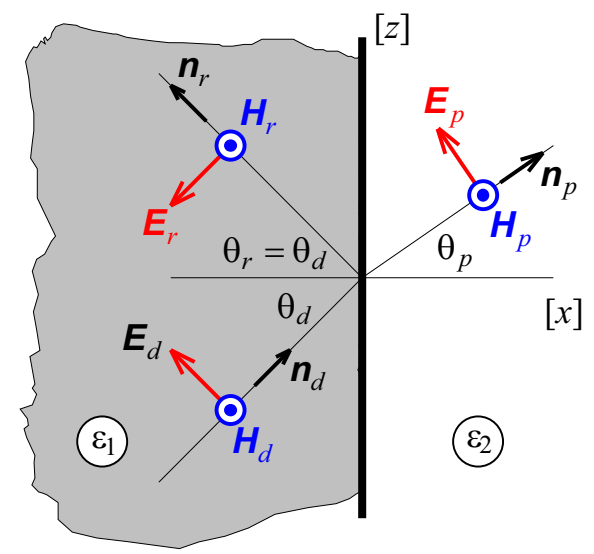

Fig. 1. TE and TM configuration of a plane wave reflection and refraction on the planar dielectric boundary

* Slovak University of Technology, Institute of Electrical Engineering Ilkovičova 3, SK-81219 Bratislava, Slovakia, lubomir.sumichrast@stuba.sk

DOI: 10.2478/jee-2019-0014, Print (till 2015) ISSN 1335-3632, On-line ISSN 1339-309X

(C) This is an open access article licensed under the Creative Commons Attribution-NonCommercial-NoDerivs License (http://creativecommons.org/licenses/by-nc-nd/3.0/). 


$$
\begin{aligned}
& \boldsymbol{E}_{r}(\boldsymbol{r})=\boldsymbol{u}_{y} E_{r} \exp \left(-j \beta_{1} \boldsymbol{n}_{r} \cdot \boldsymbol{r}\right)= \\
& =\mathbf{u}_{y} E_{r} \exp \left(j q_{1} x\right) \exp (-j k z), \\
& x<0, \\
& \boldsymbol{E}_{p}(\boldsymbol{r})=\boldsymbol{u}_{y} E_{p} \exp \left(-j \beta_{2} \mathbf{n}_{p} \cdot \mathbf{r}\right)= \\
& =\boldsymbol{u}_{y} E_{p} \exp \left(-j q_{2} x\right) \exp (-j k z), \\
& x>0,
\end{aligned}
$$

where the propagation directions of the incident, reflected and transmitted plane waves are given by the unit vectors $\mathbf{n}_{d}, \mathbf{n}_{r}, \mathbf{n}_{p}$, coplanar in the plane of incidence, where $\beta_{1}$ and $\beta_{2}$ are the phase constants of the respective media with permittivity $\varepsilon_{1}$ and $\varepsilon_{2}$ and

$$
q_{1}=\sqrt{\beta_{1}^{2}-k^{2}}=\beta_{1} \cos \theta_{d}=\beta_{1} \cos \theta_{r},
$$

$$
q_{2}=\sqrt{\beta_{2}^{2}-k^{2}}=\beta_{2} \cos \theta_{p}, k=\beta_{1} \sin \theta_{d}=\beta_{2} \sin \theta_{p} .
$$

In order to be dealing with propagating homogeneous plane waves the relation $k<\beta_{1}, \beta_{2}$ must hold.

From the above directly stem the following well-known Snellius laws of reflection and refraction

$$
\begin{gathered}
\theta_{d}=\theta_{r}, \\
\sin \theta_{p}=\sqrt{\varepsilon_{1} / \varepsilon_{2}} \sin \theta_{d} .
\end{gathered}
$$

The magnetic intensity vectors are easily obtained from the following formulae for homogeneous plane waves

$$
\begin{gathered}
\boldsymbol{H}_{d}(\boldsymbol{r})=\boldsymbol{n}_{d} \times \boldsymbol{E}_{d} \mathbf{r} / Z_{1}, \quad \boldsymbol{H}_{r}(\boldsymbol{r})=\boldsymbol{n}_{r} \times \boldsymbol{E}_{r}(\boldsymbol{r}) / Z_{1}, \\
\boldsymbol{H}_{p}(\boldsymbol{r})=\boldsymbol{n}_{p} \times \boldsymbol{E}_{p}(\boldsymbol{r}) / Z_{2},
\end{gathered}
$$

where $Z_{1}=\sqrt{\mu / \varepsilon_{1}}, Z_{2}=\sqrt{\mu / \varepsilon_{2}}$ are the wave impedances of the respective media.

The condition of continuity of tangential components of the $\boldsymbol{E}$ and $\boldsymbol{H}$ vectors on the boundary $x=0$, yields two equations for three constants $E_{d}, E_{r}$ and $E_{p}$. Any two of them can be expressed by means of the third one. Customary the reflection and transmission factors $\rho^{\mathrm{TE}}$ and $\tau^{\mathrm{TE}}$ are used expressing $E_{r}$ and $E_{p}$ by $E_{d}$, ie $E_{r}=\rho^{\mathrm{TE}} E_{d}, E_{p}=\tau^{\mathrm{TE}} E_{d}$, where

$$
\rho^{\mathrm{TE}}=\frac{E_{r}}{E_{d}}=\frac{q_{1}-q_{2}}{q_{1}+q_{2}}, \tau^{\mathrm{TE}}=\frac{E_{p}}{E_{d}}=\frac{2 q_{1}}{q_{1}+q_{2}},
$$

For the TM configuration (Fig. 1) the role of $\boldsymbol{E}$ and $\boldsymbol{H}$ vectors is interchanged, the $\boldsymbol{H}$ vectors are tangential to the boundary (in direction of $y$-axis) fulfilling equations identical with (1) - (3), the $\boldsymbol{E}$ vectors are determined from

$$
\begin{aligned}
& \boldsymbol{E}_{d}(\boldsymbol{r})=-\boldsymbol{n}_{d} \times Z_{1} \boldsymbol{H}_{d}(\boldsymbol{r}), \\
& \boldsymbol{E}_{r}(\boldsymbol{r})=-\mathbf{n}_{r} \times Z_{1} \boldsymbol{H}_{r}(\boldsymbol{r}), \\
& \boldsymbol{E}_{p}(\boldsymbol{r})=-\mathbf{n}_{p} \times Z_{2} \boldsymbol{H}_{p}(\boldsymbol{r}),
\end{aligned}
$$

and the continuity conditions of the tangential components on the boundary $x=0$ lead to the formulae

$$
\begin{gathered}
\rho^{\mathrm{TM}}=\frac{H_{r}}{H_{d}}=\frac{\varepsilon_{2} q_{1}-\varepsilon_{1} q_{2}}{\varepsilon_{2} q_{1}+\varepsilon_{1} q_{2}}, \\
\tau^{\mathrm{TM}}=\frac{H_{p}}{H_{d}}=\frac{2 \varepsilon_{2} q_{1}}{\varepsilon_{2} q_{1}+\varepsilon_{1} q_{2}} .
\end{gathered}
$$

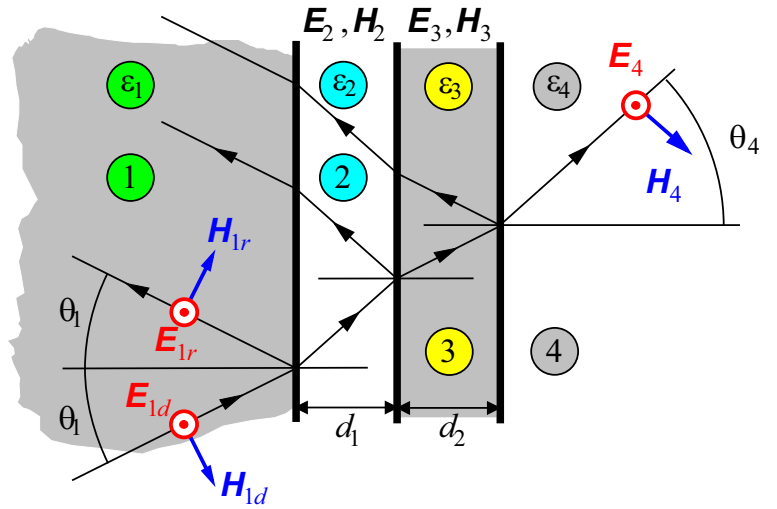

Fig. 2. Dielectric boundary frustrated by a presence of a dielectric slab (TE configuration)

Let us now consider the wave in TE configuration incident on the boundary of the structure in Fig. 2, where a dielectric slab of the thickness $d_{2}$ is placed in distance $d_{1}$ from the boundary. The seven electric-field-vectors in the respective domains of Fig. $2, x<0,0<x<d_{1}$, $d_{1}<x<d_{1}+d_{2}$ and $x>d_{1}+d_{2}$, are defined as functions of $(x, z)$

$$
\boldsymbol{E}_{1 d, 1 r}=\mathbf{u}_{y} E_{1 d, 1 r} \exp \left(\mp p j q_{1} x\right) \exp -j k z,
$$

$$
\begin{aligned}
& \boldsymbol{E}_{2 d, 2 r}=\mathbf{u}_{y} E_{2 d, 2 r} \exp \left(\mp j q_{2}\left[x-d_{1}\right]\right) \exp (-j k z), \\
& \boldsymbol{E}_{3 d, 3 r}=\mathbf{u}_{y} E_{3 d, 3 r} \exp \left(\mp j q_{3}\left[x-d_{1}-d_{2}\right]\right) \exp (-j k z), \\
& \boldsymbol{E}_{4}=\boldsymbol{u}_{y} E_{4} \exp \left(-j q_{4}\left[x-d_{1}-d_{2}\right]\right) \exp (-j k z),
\end{aligned}
$$

with

$$
\begin{gathered}
k=\beta_{1} \sin \theta_{1}=\beta_{2} \sin \theta_{2}=\beta_{3} \sin \theta_{3}=\beta_{4} \sin \theta_{4}, \\
q_{1}=\beta_{1} \cos \theta_{1}, \quad q_{2}=\beta_{2} \cos \theta_{2} \ldots \text { etc }
\end{gathered}
$$

From the six continuity conditions for tangential components of the $\boldsymbol{E}$ and $\boldsymbol{H}$ vectors on the boundaries $x=0, x=d_{1}$ and $x=d_{1}+d_{2}$, for the seven unknowns $E_{1 d}, E_{1 r}, E_{2 d}, \ldots, E_{4}$, one can express arbitrary six $E$-components by the seventh one, eg expressing six components $E_{1 d}, E_{1 r}, E_{2 d}, \ldots$, by $E_{4}$, yields for the first few terms

$$
\begin{aligned}
& E_{3 r}=E_{4} \frac{q_{3}-q_{4}}{2 q_{3}}, \quad E_{3 d}=E_{4} \frac{q_{3}+q_{4}}{2 q_{3}}, \\
& E_{2 r}=\frac{E_{4}}{2^{2} q_{2} q_{3}}\left\{\left[\left(q_{2}-q_{3}\right)\left(q_{3}+q_{4}\right)\right] \exp \left(j q_{3} d_{2}\right)+\right. \\
& \left.+\left[\left(q_{2}+q_{3}\right)\left(q_{3}-q_{4}\right)\right] \exp \left(-j q_{3} d_{2}\right)\right\}, \\
& E_{2 d}=\frac{E_{4}}{2^{2} q_{2} q_{3}}\left\{\left[\left(q_{2}+q_{3}\right)\left(q_{3}+q_{4}\right)\right] \exp \left(j q_{3} d_{2}\right)+\right. \\
& \left.+\left[\left(q_{2}-q_{3}\right)\left(q_{3}-q_{4}\right)\right] \exp \left(-j q_{3} d_{2}\right)\right\},
\end{aligned}
$$

etc, with quickly growing number of terms in each step. For TM configuration one has to proceed analogously. 


\section{Total internal reflection}

Consider for the single boundary the case $\varepsilon_{1}>\varepsilon_{2}$ and $\beta_{1}>k>\beta_{2}$. While it still holds that $\beta_{1}=\sqrt{k^{2}+q_{1}^{2}}$, to have $\beta_{2}=\sqrt{k^{2}+q_{2}^{2}}$ one has to take imaginary value of $q_{2}=-j \varkappa$ resulting in $\beta_{2}=\sqrt{k^{2}-\varkappa^{2}}$.

If the incident wave impinges upon the boundary with the angle of incidence $\theta_{d}, \theta_{d}=\arcsin \sqrt{\varepsilon_{2} / \varepsilon_{1}}$, then (7) yields $\sin \theta_{p}=1$, ie refracted wave propagates in direction parallel with the boundary. This is the definition of the critical angle $\theta_{c}$, ie

$$
\theta_{c}=\arcsin \sqrt{\varepsilon_{2} / \varepsilon_{1}} .
$$

If the wave impinges upon the boundary with the incidence angle larger than critical, $\theta_{d}>\theta_{c}$, then

$$
\sin \theta_{p}=\sin \theta_{d} / \sin \theta_{c}>1
$$

holds. This can be mathematically resolved by introducing the complex angle

$$
\theta_{p}=\pi / 2+j \varsigma
$$

Then

$$
\sin \theta_{p}=\sin (\pi / 2+j \varsigma)=\cosh \varsigma=\sin \theta_{d} / \sin \theta_{c}>1,
$$

and $\cos \theta_{p}$ becomes purely imaginary

$$
\cos \theta_{p}=\cos (\pi / 2+j \varsigma)=-j \sinh \varsigma .
$$

It also means that

$$
\begin{aligned}
& k=\beta_{2} \cosh \varsigma=\beta_{1} \sin \theta_{d}, \\
& q_{2}=-j \beta_{2} \sinh \varsigma=-j \varkappa,
\end{aligned}
$$

and

$$
\varkappa=\sqrt{k^{2}-\beta_{2}^{2}}=\beta_{1} \sqrt{\sin ^{2} \theta_{d}-\sin ^{2} \theta_{c}} .
$$

$i e$ the same result as above.

For the reflection and transmission factors in the TEcase one obtains instead of (8)

$$
\begin{gathered}
\rho^{\mathrm{TE}}=\frac{E_{r}}{E_{d}}=\frac{q+j \varkappa}{q-j \varkappa}=\exp \left(j 2 \Psi^{\mathrm{TE}}\right), \\
\tau^{\mathrm{TE}}=E_{p} / E_{d}=1+\rho^{\mathrm{TE}}=2 \cos \left(\Psi^{\mathrm{TE}}\right) \exp \left(j \Psi^{T E}\right),
\end{gathered}
$$

where for the sake of brevity, the index 1 in $q_{1}$ (4) has been omitted in (18). For the phase angle $\Psi^{\mathrm{TE}}$ simple relations hold reading

$$
\tan \Psi^{\mathrm{TE}}=\varkappa / q, \quad \cos \Psi^{\mathrm{TE}}=\cos \theta_{d} / \cos \theta_{c} .
$$

Thus the complex reflection coefficient has the magnitude $\left|\rho^{\mathrm{TE}}\right|=1$, and the phase $2 \Psi^{\mathrm{TE}}$, ie in the course of the total reflection the wave amplitude is not changed and the phase acquires the shift equal to $2 \Psi^{\mathrm{TE}}$.
The overall $E$-field in domain $x<0$, in the medium with $\varepsilon_{1}, \boldsymbol{E}_{1}=\boldsymbol{E}_{d}+\boldsymbol{E}_{r}=\boldsymbol{u}_{y} E_{1}$ is a non-homogeneous plane wave with a cosinusoidal amplitude profile propagating in the direction of $z$-axis, ie

$$
E_{1}=2 E_{d} \cos \left(q x+\Psi^{\mathrm{TE}}\right) \exp \left\{-j\left(k z-\Psi^{\mathrm{TE}}\right)\right\},
$$

while the $E$-field $\boldsymbol{E}_{2}=\boldsymbol{E}_{p}=\boldsymbol{u}_{y} E_{2}$ in second domain $x>0$, in the medium with $\varepsilon_{2}$, becomes the so-called "evanescent wave", see Fig. 3, with exponentially attenuated profile in $x$-direction

$$
E_{2}=2 E_{d} \cos \Psi^{\mathrm{TE}} \exp (-\varkappa x) \exp \left\{-j\left(k z-\Psi^{\mathrm{TE}}\right)\right\},
$$

both acquiring the phase shift $\Psi^{\mathrm{TE}}$ in direction of propagation (Fig. 3).

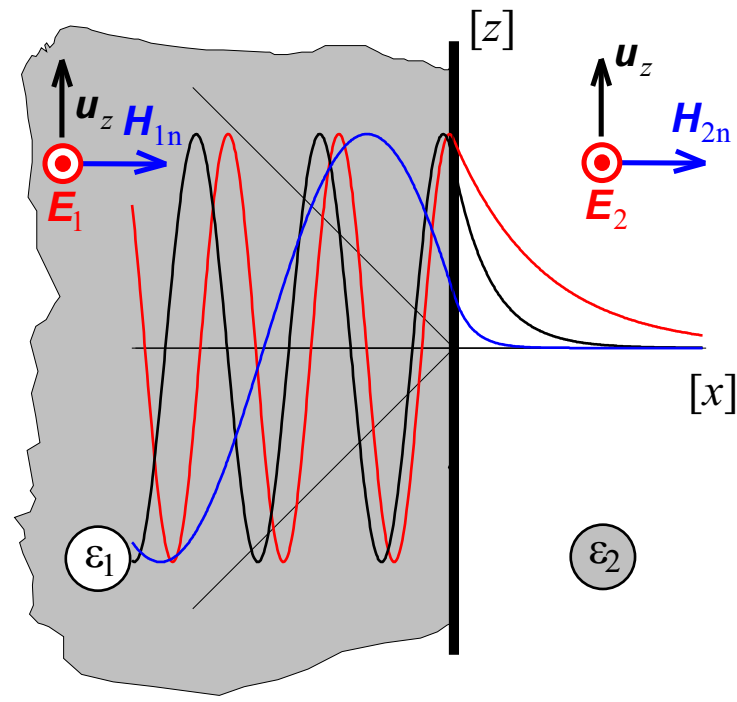

Fig. 3. Schematics of the total reflection and overall wave profile for $\theta_{c}=10^{\circ}$, and for $\theta_{d}=15^{\circ}$ (red), $\theta_{d}=30^{\circ}$ (black), $\theta_{d}=75^{\circ}$ (blue)

In the TM-case the reflection and transmission factors $\rho^{\mathrm{TM}}$ and $\tau^{\mathrm{TM}}$ are defined for $\boldsymbol{H}$ vectors, $H_{r}=\rho^{\mathrm{TM}} H_{d}$, $H_{p}=\tau^{\mathrm{TM}} H_{d}$, resulting in

$$
\begin{aligned}
& \rho^{\mathrm{TM}}=\frac{q \varepsilon_{2}+j \varkappa \varepsilon_{1}}{q \varepsilon_{2}-j \varkappa \varepsilon_{1}}=\frac{q \sin ^{2} \theta_{c}+j \varkappa}{q \sin ^{2} \theta_{c}-j \varkappa}= \\
& =\exp \left(j 2 \Psi^{\mathrm{TM}}\right),
\end{aligned}
$$

with

$$
\tan \Psi^{\mathrm{TM}}=\varkappa / q \sin ^{2} \theta_{c}=\tan \Psi^{\mathrm{TE}} / \sin ^{2} \theta_{c}
$$

and with the same expression for $\tau^{\mathrm{TM}}$ as (19), $\tau^{\mathrm{TM}}=$ $1+\rho^{\mathrm{TM}}=2 \cos \left(\Psi^{\mathrm{TM}}\right) \exp \left(j \Psi^{\mathrm{TM}}\right)$.

In both, the TE as well as in the TM configuration, for the wave incident upon the critical angle $\theta_{d}=\theta_{c}$, the reflection coefficients are equal to one, $\rho=1$ and $\Psi=0$. For the limiting case of $\theta_{d}=\pi / 2$ (so called "grazing incidence"), $\rho=-1$ and $\Psi=\pi / 2$. For the TM configuration is the phase angle $\Psi^{\mathrm{TM}}$ always larger than the phase angle $\Psi^{\mathrm{TE}}$ for the TE configuration, as it is clearly seen in Fig. 4. 


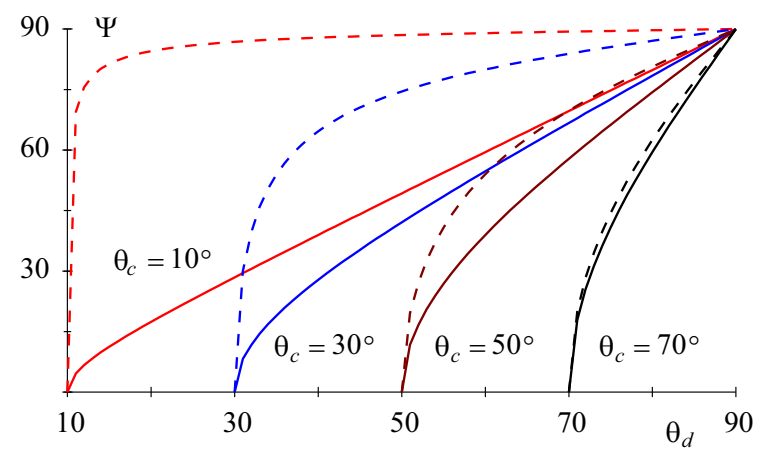

Fig. 4. Dependence of phase shift $\Psi$ on the angle-of incidence $\theta_{d}$ (in degrees, full line for TE the, dashed line for TM configuration)

\section{Total internal reflection frustrated by a dielectric slab}

Let us consider the structure as in Fig. 2. with $\varepsilon_{3}=\varepsilon_{1}$ in domain $3, d_{1}<x<d_{1}+d_{2}$, and $\varepsilon_{4}=\varepsilon_{2}$ in domain 4, $x>d_{1}+d_{2}$, provided $\varepsilon_{1}>\varepsilon_{2}$, and the wave incident on the boundary $1-2, x=0$, with angle $\theta_{1}=\theta_{d}$ larger than the critical angle $\theta_{c}=\sqrt{\varepsilon_{2} / \varepsilon_{1}}$.

The electric-field-vectors as functions of $(x, z)$ in respective domains are defined as

$$
\begin{gathered}
\boldsymbol{E}_{1 d, 1 r}=\mathbf{u}_{y} E_{1 d, r} \exp (\mp j q x) \exp (-j k z), \\
\boldsymbol{E}_{2 d, 2 r}=\mathbf{u}_{y} E_{2 d, r} \exp \left\{\mp \varkappa\left(x-d_{1}\right)\right\} \exp (-j k z), \\
\boldsymbol{E}_{3 d, 3 r}=\mathbf{u}_{y} E_{3 d, r} \exp \left\{\mp j q\left(x-d_{1}-d_{2}\right)\right\} \exp (-j k z), \\
\boldsymbol{E}_{4}=\mathbf{u}_{y} E_{4} \exp \left\{-\varkappa\left(x-d_{1}-d_{2}\right)\right\} \exp (-j k z),
\end{gathered}
$$

$i e$ as the homogeneous plane waves in domains 1 and 3 and the evanescent waves in domains 2 and 4 , where $q$ and $\varkappa$ are given by (4) and (17).

For the seven unknowns $E_{1 d}, E_{1 r}, E_{d 2}, \ldots, E_{4}$, one can express arbitrary six $E$-components by the seventh one. Expressing six components $E_{1 d}, E_{1 r}, E_{d 2}, \ldots, E_{3 r}$ by $E_{4}$ from the six continuity conditions for tangential components of the $\boldsymbol{E}$ and $\boldsymbol{H}$ vectors on the boundaries $x=0, x=d_{1}$ and $x=d_{1}+d_{2}$ yields

$$
\begin{gathered}
E_{3 r}=E_{4} \frac{\exp (j \Psi)}{2 \cos (\Psi)}, \quad E_{3 d}=E_{4} \frac{\exp (-j \Psi)}{2 \cos (\Psi)} \\
E_{2 d}=E_{4} \frac{\sin \left(2 \Psi-q d_{2}\right)}{\sin (2 \Psi)}, \quad E_{2 r}=E_{4} \frac{\sin \left(q d_{2}\right)}{\sin (2 \Psi)} \\
E_{1 d}=E_{4} \frac{A \exp (-j \Psi)+B \exp (j \Psi)}{2 \cos (\Psi) \sin (2 \Psi)} \\
E_{1 r}=E_{4} \frac{A \exp (j \Psi)+B \exp (-j \Psi)}{2 \cos (\Psi) \sin (2 \Psi)}
\end{gathered}
$$

where $\Psi=\Psi^{\mathrm{TE}}$ as in (20), and

$$
\begin{aligned}
& A=\sin \left(2 \Psi-q d_{2}\right) \exp \left(\varkappa d_{1}\right) \\
& B=\sin \left(q d_{2}\right) \exp \left(-\varkappa d_{1}\right)
\end{aligned}
$$

For the TM configuration the formulae (29) through (33) remain the same for $H_{1 d}, H_{1 r}, H_{d 2}, \ldots, H_{3 r}$ expressed by $H_{4}$, and for $\Psi=\Psi^{\mathrm{TM}}$ as in (24).

Defining reflection factors on the respective boundaries yields

$$
\begin{gathered}
\rho_{34}=\exp (j 2 \Psi), \quad \rho_{23}=\frac{\sin \left(q d_{2}\right)}{\sin \left(2 \Psi-q d_{2}\right)}, \\
\rho_{12}=\frac{A \exp (j \Psi)+B \exp (-j \Psi)}{A \exp (-j \Psi)+B \exp (j \Psi)}=\exp (j 2 \Theta), \\
\tan \Theta=\frac{A-B}{A+B} \tan \Psi,
\end{gathered}
$$

for both TE and TM configuration respectively.

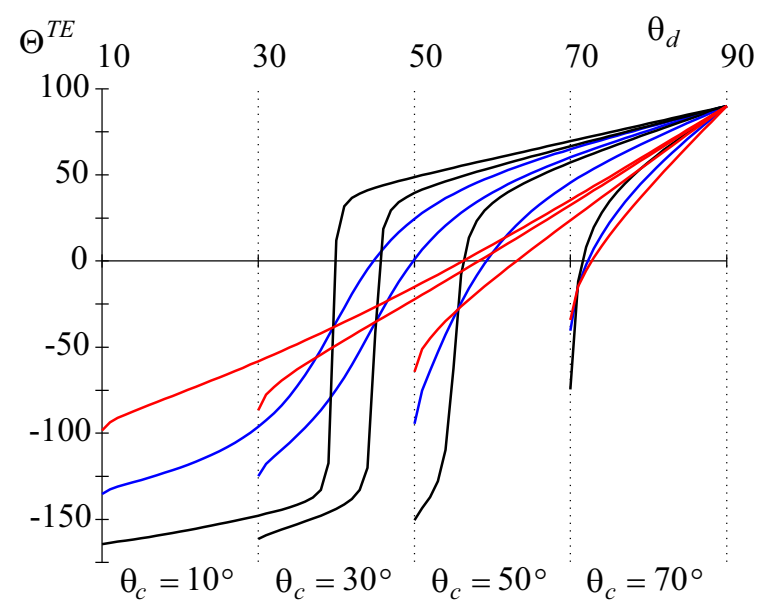

Fig. 5. Dependence of the phase shift $\Theta^{\mathrm{TE}}$ on the angle-of incidence $\theta_{d}$ for $\beta_{1} d_{2}=100^{\circ}, d_{1}=0$, (red), $d_{1} / d_{2}=0.5$, (blue), $d_{1} / d_{2}=2$, (black), and the same critical angles $\theta_{c}$ as in Fig. 4

On the boundary $3-4, x=d_{1}+d_{2}$, the reflection factor $\rho_{34}$ in (34) is in fact the same as on the single boundary (18). On the boundary $1-2, x=0$, the magnitude of the reflection factor $\rho_{12}$ equals to one and the phase angle $\Theta$ given by (36) is depicted in Figs. 5 and 6 . This dependance is, similarly as in the case of the single boundary, always monotonically increasing. Note the substantial differences in curve shapes between the TE and TM case.

The maximum value of $\Theta=\pi / 2$ is reached for the grazing incidence $\theta_{d}=\pi / 2$ and the minimum value is reached for the wave incidence upon the critical angle $\theta_{d}=\theta_{c}$, ie as the limiting case of (35) for $\Psi \rightarrow 0$ yielding

$$
\left.\rho_{12}^{\mathrm{TE}}\right|_{\Psi=0}=\frac{\exp \left(-j q d_{2}\right)-q d_{1} \sin \left(q d_{2}\right)}{\exp \left(j q d_{2}\right)-q d_{1} \sin \left(q d_{2}\right)}
$$




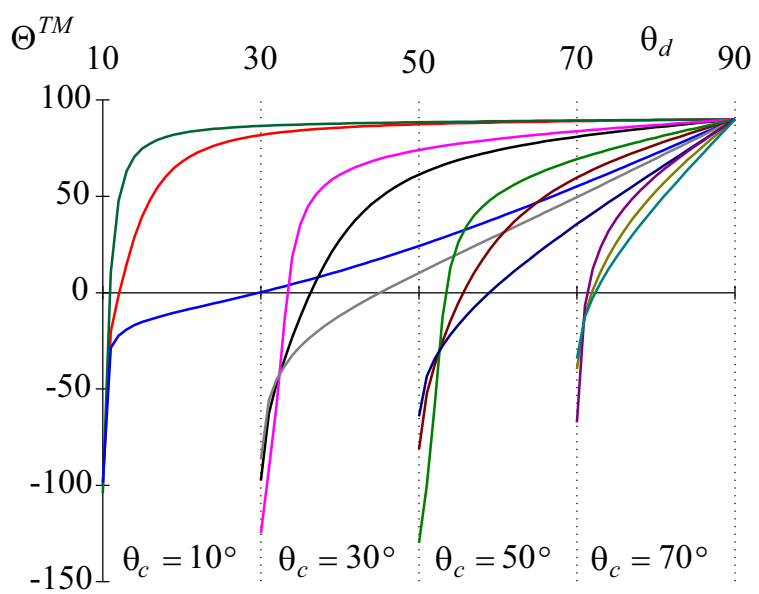

Fig. 6. Dependence of the phase shift $\Theta^{\mathrm{TM}}$ on the angle-of incidence $\theta_{d}$ for $\beta_{1} d_{2}=100^{\circ}, d_{1}=0,(\mathrm{red}), d_{1} / d_{2}=0.5$ (blue), $d_{1} / d_{2}=2$ (black), and the same critical angles $\theta_{c}$ as in Fig. 4

$$
\left.\rho_{12}^{\mathrm{TM}}\right|_{\Psi=0}=\frac{\exp \left(-j q d_{2}\right)-q d_{1} \sin ^{2} \theta_{c} \sin \left(q d_{2}\right)}{\exp \left(j q d_{2}\right)-q d_{1} \sin ^{2} \theta_{c} \sin \left(q d_{2}\right)} .
$$

For $d_{1}=0$, ie for the zero gap between the bulk domain of $\varepsilon_{1}$ and the dielectric slab of the same permittivity, one obtains from (35)

$$
\rho_{12}=\exp \left\{j 2\left(\Psi-q d_{2}\right)\right\} .
$$

This is a self-evident result since this case represents just the simple boundary case, comparing the incident and the reflected wave in depth of the $\varepsilon_{1}$ domain, in distance $d_{2}$ from the boundary. The result must be the phase shift of $2 \Psi$ due to total reflection, together with the phase shift $-2 q d_{2}$, ie twice the wave phase shift in $x$-direction along length $d_{2}$ - to the boundary and back.

For $d_{1} \rightarrow \infty$ (36) yields $\Theta=\Psi$, ie an infinitely distant dielectric slab of course has no influence on the reflected wave.

For $\sin \left(2 \Psi-q d_{2}\right)=0$, ie $q d_{2}=2 \Psi+n \pi$, one gets, see (33), $A=0$, and (36) yields value of $\Theta$ independent from $d_{1}$, equal to $\Theta=-\Psi$. These are just the intersection points of red, blue and black curves in Fig. 5 and 6 for particular values of $\theta_{c}$ equal to 10, 30, 50 and 70 degrees respectively. Observe that for this condition $E_{2 d}$ in (30) (and analogously $H_{2 d}$ for TM configuration) equals to zero, ie the exponentially decreasing term $\boldsymbol{E}_{2 d}(x, z)=$ $\ldots \exp \left(\varkappa\left[x-d_{1}\right]\right) \ldots$ in the domain $x \in\left(0, d_{1}\right)$ does not exist, only the exponentially increasing term $\boldsymbol{E}_{2 r}(x, z)=$ $\ldots \exp \left(\varkappa\left[x-d_{1}\right]\right) \ldots$ plays a role.

The condition $q d_{2}=2 \Psi+n \pi$ can be recast into the form of two equations $q \tan \left(q d_{2} / 2\right)=\varkappa$ and $\sqrt{q^{2}+\varkappa^{2}}=$ $\sqrt{\beta_{1}^{2}-\beta_{2}^{2}}$, for two variables $q$ and $\varkappa$. This two equations represent exactly the condition for the existence of a waveguide mode in dielectric slab waveguide of thickness $d_{2}$ with permittivity $\varepsilon_{1}$, surrounded by the infinite medium of permittivity $\varepsilon_{2}$.

If $\sin \left(2 \Psi-q d_{2}\right) \exp \left(\varkappa d_{1}\right)=\sin \left(q d_{2}\right) \exp \left(-\varkappa d_{1}\right)$, ie if $A=B$ accordingly (33), then (36) yields $\Theta=0, \rho_{12}=1$.
These are the intersection points of the curves in Fig. 5 and 6 with the horizontal axis.

\section{Goos-Hänchen shift of a non-homogeneous plane wave with the cos-like amplitude pattern}

Let us now consider an interference of two incident homogeneous TE-polarised plane waves propagating in directions given by $\mathbf{n}_{d A}$ and $\boldsymbol{n}_{d B}$ as in Fig. 7

$$
\begin{aligned}
\boldsymbol{E}_{d}(\boldsymbol{r}) & =\mathbf{u}_{y} E_{0} \exp \left(-j \beta_{1} \mathbf{n}_{d A} \cdot \boldsymbol{r}\right) \\
& +\mathbf{u}_{y} E_{0} \exp \left(-j \beta_{1} \mathbf{n}_{d B} \cdot \boldsymbol{r}\right),
\end{aligned}
$$

$\mathbf{n}_{d A}=\cos \theta_{A} \boldsymbol{u}_{x}+\sin \theta_{A} \boldsymbol{u}_{z}, \mathbf{n}_{d B}=\cos \theta_{B} \boldsymbol{u}_{x}+\sin \theta_{B} \boldsymbol{u}_{z}$ assuming $\theta_{A}>\theta_{B}$.

Introducing two perpendicular non-unit vectors $\boldsymbol{n}_{d 0}$ and $\boldsymbol{n}_{A B}$ (Fig. 7) by

$$
\begin{gathered}
\boldsymbol{n}_{d 0}=\left(\boldsymbol{n}_{d A}+\mathbf{n}_{d B}\right) / 2= \\
=\cos \theta_{A B}\left(\cos \theta_{d 0} \boldsymbol{u}_{x}+\sin \theta_{d 0} \boldsymbol{u}_{z}\right), \\
\boldsymbol{n}_{A B}=\left(\boldsymbol{n}_{d A}-\boldsymbol{n}_{d B}\right) / 2= \\
=\sin \theta_{A B}\left(-\sin \theta_{d 0} \boldsymbol{u}_{x}+\cos \theta_{d 0} \boldsymbol{u}_{z}\right), \\
\theta_{d 0}=\left(\theta_{A}+\theta_{B}\right) / 2, \quad \theta_{A B}=\left(\theta_{A}-\theta_{B}\right) / 2,
\end{gathered}
$$

one may rewrite (40) as

$$
\boldsymbol{E}_{d}(\boldsymbol{r})=\boldsymbol{u}_{y} 2 E_{0} \cos \left(\beta_{1} \boldsymbol{n}_{A B} \cdot \boldsymbol{r}\right) \exp \left(-j \beta_{1} \boldsymbol{n}_{d 0} \cdot \boldsymbol{r}\right)
$$

$i e$ as an inhomogeneous plane having in the transversal direction $\mathbf{n}_{A B}$, perpendicular to the propagation direction $\mathbf{n}_{d 0}$, a non-constant, cosinusoidal amplitude pattern of the wavefront

$$
\begin{aligned}
& 2 E_{0} \cos \left(\beta_{1} \boldsymbol{n}_{A B} \cdot \boldsymbol{r}\right)= \\
= & 2 E_{0} \cos \left\{\beta_{1} \sin \theta_{A B}\left(-x \sin \theta_{d 0}+z \cos \theta_{d 0}\right)\right\}= \\
= & 2 E_{0} \cos \left\{q_{A B} \xi\right\},
\end{aligned}
$$

with the spatial frequency $q_{A B}=\beta_{1} \sin \theta_{A B}$ and transversal variable $\xi$. This non-homogeneous plane wave propagates in direction $\boldsymbol{n}_{d 0}$ with the propagation factor $\exp \left(-j \beta_{1} \boldsymbol{n}_{d 0} \cdot \boldsymbol{r}\right)$ equal to

$$
\begin{array}{r}
\exp \left\{-j \beta_{1} \cos \theta_{A B}\left(x \cos \theta_{d 0}+z \sin \theta_{d 0}\right)\right\} \\
=\exp \left\{-j k_{A B} \zeta\right\}
\end{array}
$$

thus propagating with the wavenumber $k_{A B}=\beta_{1} \cos \theta_{A B}$ along the longitudinal variable $\zeta$.

If both directions $\boldsymbol{n}_{d A}$ and $\boldsymbol{n}_{d B}$ fulfil the condition of the total reflection $\theta_{A}, \theta_{B}>\theta_{c}$, then for the resulting reflected wave one obtains

$$
\begin{aligned}
& \boldsymbol{E}_{r}(\boldsymbol{r})=\mathbf{u}_{y} E_{0}\left\{\exp \left(2 j \Psi_{A}\right) \exp \left(-j \beta_{1} \mathbf{n}_{r A} \cdot \boldsymbol{r}\right)+\right. \\
& \left.+\exp \left(2 j \Psi_{B}\right) \exp \left(-j \beta_{1} \mathbf{n}_{r B} \cdot \boldsymbol{r}\right)\right\}
\end{aligned}
$$




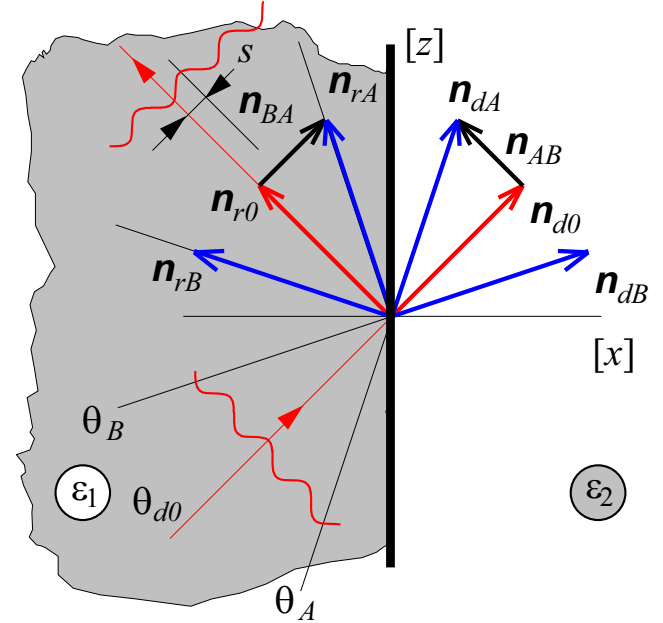

Fig. 7. Definition of the vectors $\boldsymbol{n}_{d A}, \boldsymbol{n}_{d B}, \boldsymbol{n}_{d 0}, \boldsymbol{n}_{r A}, \boldsymbol{n}_{r B}, \boldsymbol{n}_{r 0}$, $\boldsymbol{n}_{A B}$ and $\boldsymbol{n}_{B A}$, and of the pertaining angles $\theta_{A}, \theta_{B}, \theta_{d 0}$, together with the schematic picture of the Goos-Hänchen shift $s$ for the plane wave with the cosinusoidal amplitude pattern

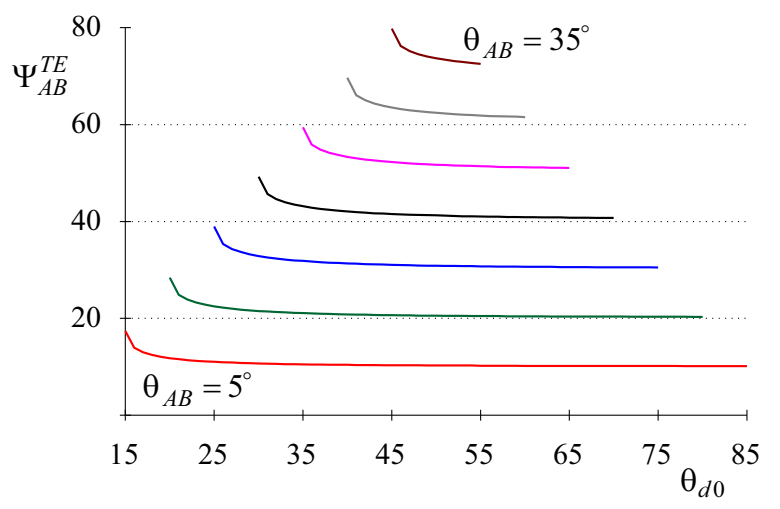

Fig. 8. Single-boundary GH-shift-phase $\Psi_{A B}^{\mathrm{TE}}$ in TE configuration, $\theta_{c}=10^{\circ}, \theta_{A B} \in\left\{5^{\circ}, 35^{\circ}\right\}$ in steps of $5^{\circ}$, as a function of the beam-axis angle of incidence $\theta_{d 0}$

where the reflection factors $\exp \left(j 2 \Psi_{A}\right)$ and $\exp \left(j 2 \Psi_{B}\right)$ pertain to the angles of incidence $\theta_{A}$ and $\theta_{B}$ respectively, and

$$
\begin{aligned}
& \boldsymbol{n}_{r A}=-\cos \theta_{A} \boldsymbol{u}_{x}+\sin \theta_{A} \boldsymbol{u}_{z}, \\
& \mathbf{n}_{r B}=-\cos \theta_{B} \boldsymbol{u}_{x}+\sin \theta_{B} \boldsymbol{u}_{z} .
\end{aligned}
$$

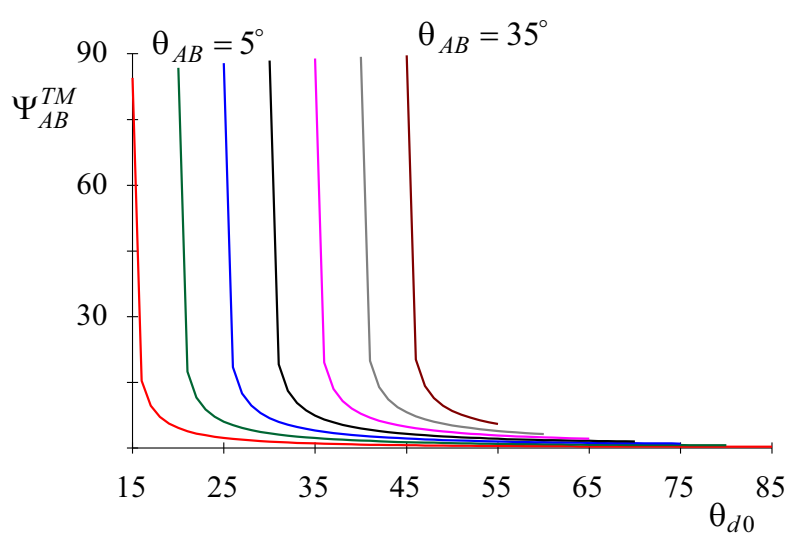

Fig. 9. The same as in Fig.8, but for the TM configuration
Denoting similarly as above

$$
\begin{aligned}
& \mathbf{n}_{r 0}=\left(\mathbf{n}_{r A}+\mathbf{n}_{r B}\right) / 2= \\
& =\cos \theta_{A B}\left(-\cos \theta_{d 0} \boldsymbol{u}_{x}+\sin \theta_{d 0} \boldsymbol{u}_{z}\right), \\
& \mathbf{n}_{B A}=\left(\mathbf{n}_{r A}-\mathbf{n}_{r B}\right) / 2= \\
& =\sin \theta_{A B}\left(\sin \theta_{d 0} \boldsymbol{u}_{x}+\cos \theta_{d 0} \boldsymbol{u}_{z}\right),
\end{aligned}
$$

formula (44) can be rewritten into the form

$$
\begin{aligned}
& \boldsymbol{E}_{r}(\boldsymbol{r})=\boldsymbol{u}_{y} 2 E_{0} \cos \left(\beta_{1} \boldsymbol{n}_{B A} \cdot \boldsymbol{r}-\Psi_{A B}\right) \times \\
& \times \exp \left\{-j\left(\beta_{1} \boldsymbol{n}_{r 0} \cdot \boldsymbol{r}-\Psi_{0}\right)\right\},
\end{aligned}
$$

where $\Psi_{0}=\Psi_{A}+\Psi_{B}$ and $\Psi_{A B}=\Psi_{A}-\Psi_{B}$.

The cosinusoidal amplitude pattern of the reflected wave along the transversal variable $\chi$ is now given by

$$
\begin{gathered}
2 E_{0} \cos \left(\beta_{1} \mathbf{n}_{B A} \cdot \mathbf{r}-\Psi_{A B}\right)= \\
=2 E_{0} \cos \left\{\beta_{1} \sin \theta_{A B}\left(\sin \theta_{d 0} x+\cos \theta_{d 0} z\right)-\Psi_{A B}\right\}= \\
=2 E_{0} \cos \left\{q_{A B} \chi-\Psi_{A B}\right\},
\end{gathered}
$$

with the same spatial frequency $q_{A B}$ as the amplitude pattern of the incident wave but shifted in the direction of $\boldsymbol{n}_{B A}$ by

$$
s=\Psi_{A B} / \beta_{1} \sin \theta_{A B} .
$$

This effect is called the Goos-Hänchen (GH) shift. The propagation factor $\exp \left\{-j\left(\beta_{1} \mathbf{n}_{r 0} \cdot \mathbf{r}-\Psi_{0}\right)\right\}$ of the reflected wave, propagating in direction of the vector $\mathbf{n}_{r 0}$, $i e$ along the longitudinal variable $\eta$,

$$
\begin{aligned}
& \exp \left\{-j\left[\beta_{1} \cos \theta_{A B}\left(-\cos \theta_{d 0} x+\sin \theta_{d 0} z\right)-\Psi_{0}\right]\right\}= \\
& =\exp \left\{-j\left(k_{A B} \eta-\Psi_{0}\right)\right\}
\end{aligned}
$$

has, due to the total reflection acquired the phase shift $\Psi_{0}$.

Exactly the same development is valid for the case of total internal reflection frustrated by a dielectric slab, just the phase angles $\Psi$ have to be substituted by phase angles $\Theta$.

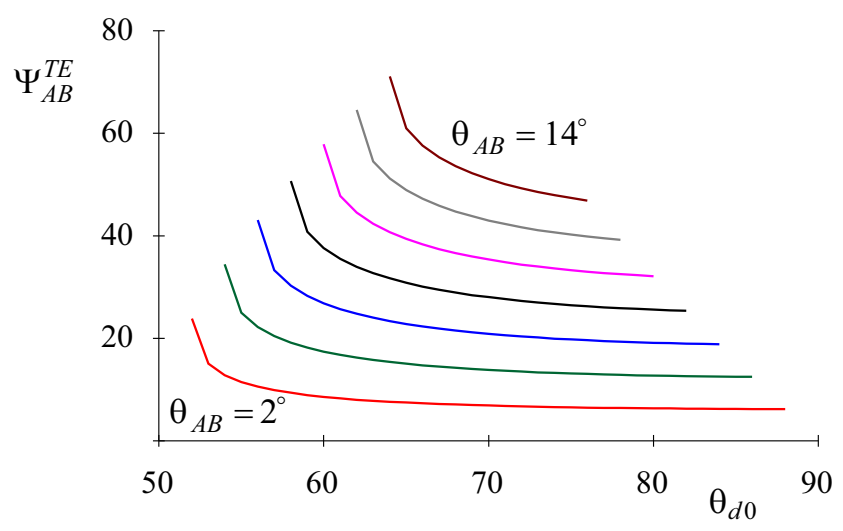

Fig. 10. Single-boundary GH-shift-phase $\Psi_{A B}^{\mathrm{TE}}$ in TE configuration, $\theta_{c}=50^{\circ}, \theta_{A B}=\left\{2^{\circ}, 14^{\circ}\right\}$ in steps of $2^{\circ}$, as a function of the beam-axis angle of incidence $\theta_{d 0}$ 


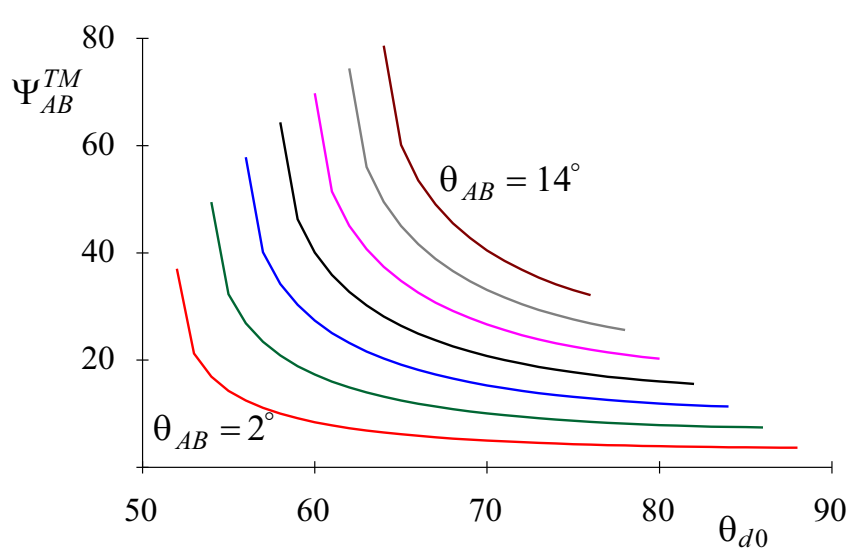

Fig. 11. The same as in fig.10, but for the TM configuration

The depedence of the GH-shift-phase-angles $\Psi_{A B}$ (or $\left.\Theta_{A B}\right)$ on the angle of incidence $\theta_{d 0}$ are depicted in Figs. 8 through 17 for different critical angles $\theta_{c}$ and different angles $\theta_{A B}$, ie for the different spatial frequencies $q_{A B}=$ $\beta_{1} \sin \theta_{A B}$ of the cosinusoidal amplitude pattern of nonhomogeneous plane wave.

As it is easily seen from Fig. 8 for TE configuration and $\theta_{c}=10^{\circ}$, doubling the angle $\theta_{A B}$ approximately doubles the value of $\Psi_{A B}^{\mathrm{TE}}$ too. Apart from the low and high end of $\theta_{d 0}$ dependance, the values of $\Psi_{A B}^{\mathrm{TE}}$ are pretty constant. This is not the case for the TM configuration having completely different character of this dependance as seen from Fig. 9.

In fact the curves of $\Psi_{A B}$ (or $\Theta_{A B}$ ) in Fig. 8 - Fig. 17 follow directly from the respective curves in Fig. 4 -Fig. 6 as a difference of values $\Psi$ (or $\Theta$ ) for two values of $\theta$, namely $\theta_{A}=\theta_{d 0}+\theta_{A B}$ and $\theta_{B}=\theta_{d 0}-\theta_{A B}$.

As seen from Fig. 10 and 11 for the critical angle $\theta_{c}=50^{\circ}$ the respective curves for TE and for TM configuration are quite similar to each other as it can be concluded also from Fig. 5 and 6 , since the respective curves there, are mutually much closer for $\theta_{c}=50^{\circ}$, than for $\theta_{c}=10^{\circ}$.

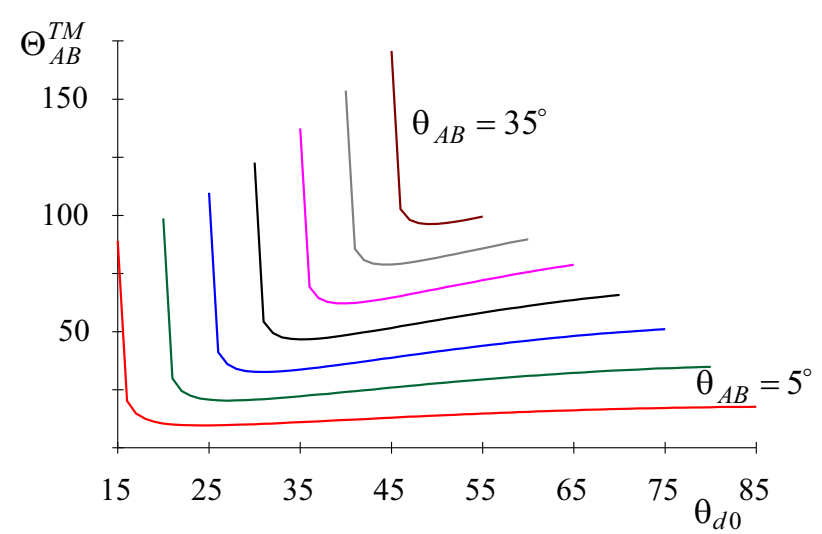

Fig. 13. The same as in fig.12, but for the TM configuration

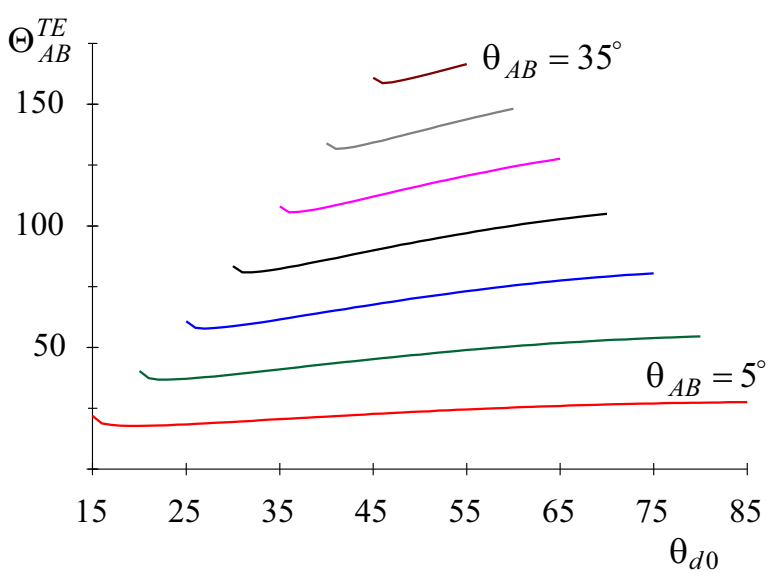

Fig. 12. Single-boundary GH-shift-phase $\Theta_{A B}^{\mathrm{TE}}$ in TE configuration, $\theta_{c}=10^{\circ}, s_{1}=0, \beta_{1} d_{2}=100^{\circ} \theta_{A B}=\left\{5^{\circ}, 35^{\circ}\right\}$ in steps of $5^{\circ}$, as a function of the beam-axis angle of incidence $\theta_{d 0}$

As already mentioned, for $d_{1}=0$ one deals with the single boundary, where the GH shift is investigated within the bulk domain in a plane shifted a distance $d_{2}$ from the boundary. For this case one obtains from (39) the relation

$$
\Theta_{A B}=\Psi_{A B}+2 \beta_{1} d_{2} \sin \theta_{d 0} \sin \theta_{A B}
$$

bounding together the curves in Fig. 8 with the curves in Fig. 12 as well as curves in Fig. 9 with curves in Fig. 13. The increased range of $\Theta_{A B}$ angles is clearly manifested.

If there is a growing gap of width $d_{1}$ between the bulk material and the dielectric slab, then the range of angles $\Theta_{A B}$ grows further and the shape of curves, particularly for TE configuration, changes significantly. However one has to keep in mind that, though the angles $\Theta_{A B}$ larger than $180^{\circ}$ indicate the GH shift larger than a half-period to the right on the $\chi$ axis, they can be equally well interpreted also as a GH shift to the left smaller than a half-period corresponding to the angle $\pi-\Theta_{A B}$.

If the gap $d_{1}$ is further increased the influence of the dielectric slab decreases and the curves in Figs. 14 through 17 gradually converge to the curves in Figs. 8 and 9 - compare eg Fig. 17 with Fig. 9.

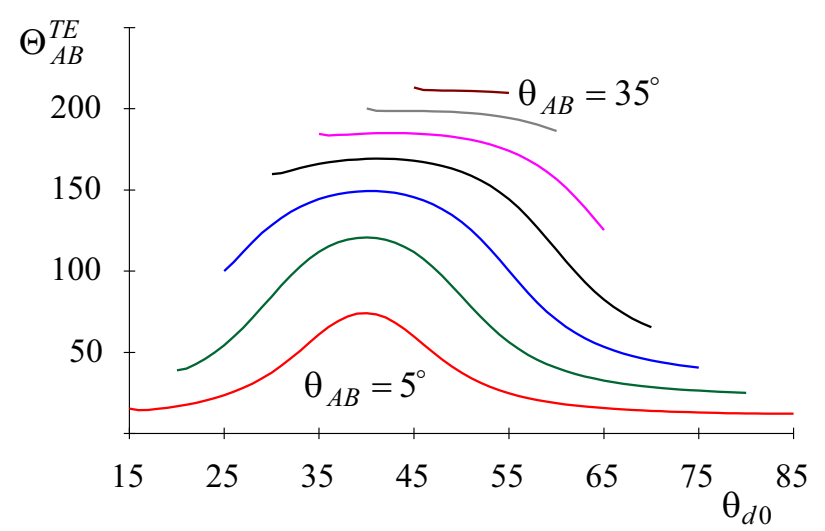

Fig. 14. Dielectric-slab-frustrated GH-shift-phase $\Theta_{A B}^{\mathrm{TE}}$, in TE configuration, $\theta_{c}=10^{\circ}, d_{1} / d_{2}=0.5, \beta_{1} d_{2}=100^{\circ}, \theta_{A B} \in$ $\left\{5^{\circ}, 35^{\circ}\right\}$ in steps of $5^{\circ}$, as a function of the beam-axis angle of incidence $\theta_{d 0}$. 


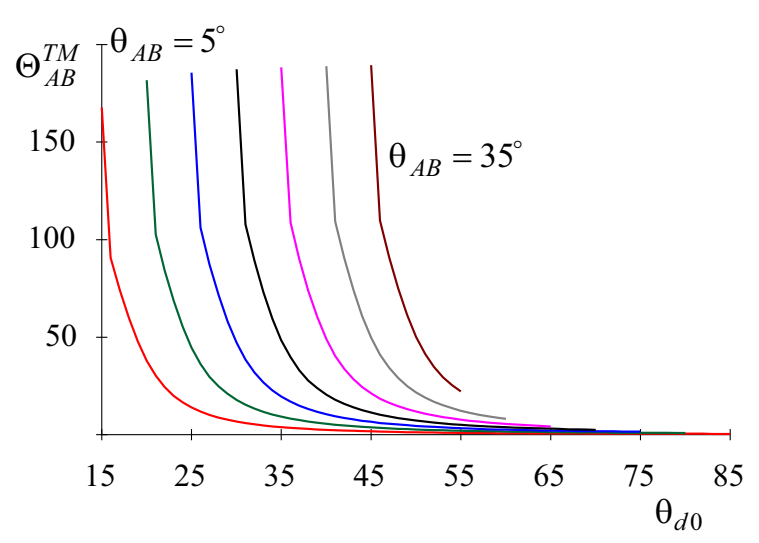

Fig. 15. The same as in fig.14, but for the TM configuration

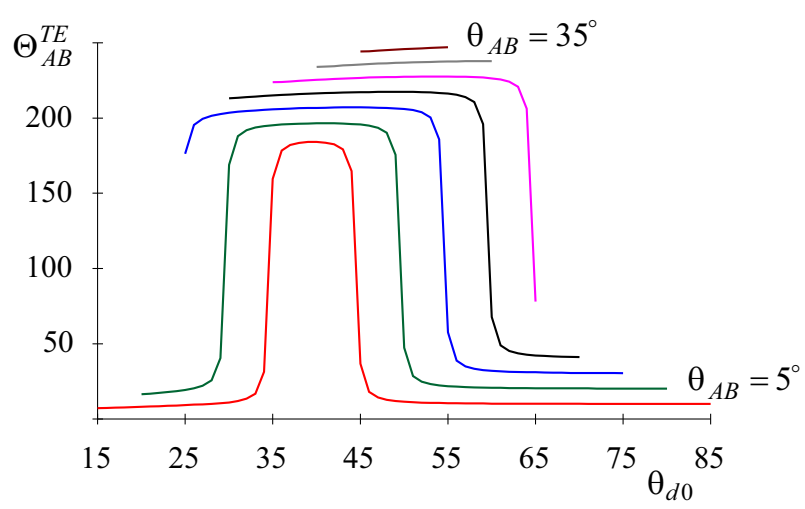

Fig. 16. Dielectric-slab-frustrated GH-shift-phase $\Theta_{A B}^{\mathrm{TE}}$, in $\mathrm{TE}$ configuration, $\theta_{c}=10^{\circ}, d_{1} / d_{2}=2, \beta_{1} d_{2}=100^{\circ}, \theta_{A B} \in\left\{5^{\circ}, 35^{\circ}\right\}$ in steps of $5^{\circ}$, as a function of the beam-axis angle of incidence $\theta_{d 0}$.

\section{Total reflection of the beam wave-beam shift and beam profile deformation}

In this section we follow the development thoroughly explained in [7]. Generally, the spatially confined beam wave can be expressed as [7]

$$
\boldsymbol{E}_{d}(\boldsymbol{r})=\boldsymbol{E}_{d}(\xi, \zeta)=\mathbf{u}_{y} E_{y}(\xi, \zeta)=\mathbf{u}_{y} E_{0} \varphi_{d}(\xi, \zeta)
$$

$i e$ as the beam wave propagating along the longitudinal $\zeta$-axis under the angle-of-incidence $\theta_{d 0}$ to the $x$-axis (see (41) through (43) and Fig. 19). The amplitude profile $\varphi_{d}(\xi, \zeta)$ of the beam wave is a finite sum of discrete spatial frequency components of type

$$
\varphi_{d n}(\xi, \zeta)=\cos \left\{q_{n} \xi\right\} \exp \left\{-j k_{n} \zeta\right\}, n=0,1, \ldots, N,
$$

with the transversal $\xi$-axis perpendicular to $\zeta$-axis, and $q_{n}=\beta_{1} \sin \theta_{n}, k_{n}=\beta_{1} \cos \theta_{n}$.

The reflected beam wave propagating along the longitudinal $\eta$ is given analogously as

$$
\boldsymbol{E}_{r}(\boldsymbol{r})=\boldsymbol{E}_{r}(\chi, \eta)=\mathbf{u}_{y} E_{y}(\chi, \eta)=\mathbf{u}_{y} E_{0} \varphi_{r}(\chi, \eta)
$$

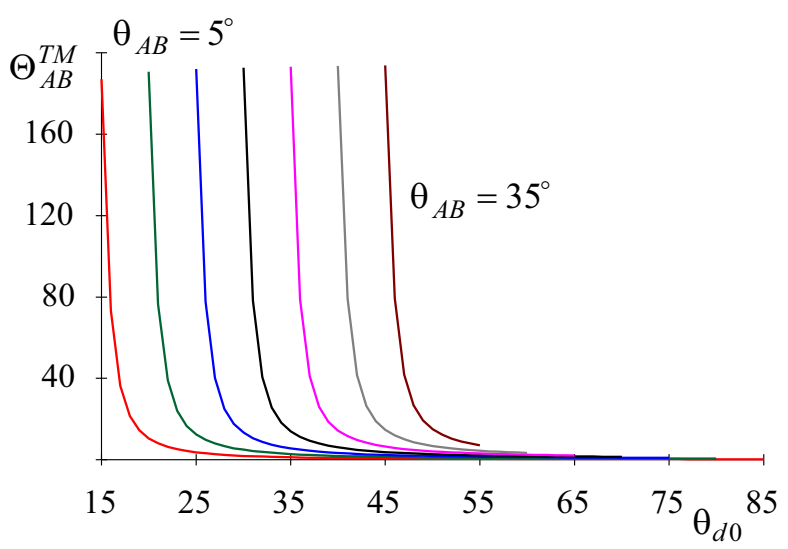

Fig. 17. The same as in fig.16, but for the TM configuration

where $\varphi_{r}(\chi, \zeta)$ is a finite sum of discrete spatial frequency components of the type

$$
\varphi_{r n}(\chi, \zeta)=\cos \left\{q_{n} \chi-\Psi_{n}\right\} \exp \left\{-j\left(k_{n} \zeta-\Psi_{0 n}\right)\right\}
$$

where $\Psi_{n}=\Psi_{A n}-\Psi_{B n}, \Psi_{0 n}=\Psi_{A n}+\Psi_{B n}$ and $\Psi_{A n}$, $\Psi_{B n}$ are the total-reflection-phase-shifts accordingly (20) (for TE configuration, or (24) for TM configuration, or $\Theta_{A n}, \Theta_{B n}$ accordingly (36) in case of frustrating dielectric slab presence respectively) for the angles of incidence $\theta_{A n}=\theta_{d 0}+\theta_{n}$ and $\theta_{B n}=\theta_{d 0}-\theta_{n}$, with $\theta_{n}=\arcsin \left(q_{n} / \beta_{1}\right\}$.

In our case we have taken as the initial profile in the plane $\zeta=0$, ie in the incident-beam waist plane, the periodic function $\varphi_{d}(\xi, 0)$, with the period $\xi_{\max }$, on the interval $\left(-\xi_{\max } / 2, \xi_{\max } / 2\right)$, in the form

$$
\varphi_{d}(\xi, 0)=\frac{1}{2 N+1}+\sum_{n=1}^{N} \frac{2}{2 N+1} \cos \left(n \Delta_{q} \xi\right)
$$

leading to the electric field of the propagating beam in the form

$$
\begin{gathered}
\boldsymbol{E}_{d}(\xi, \zeta)=\mathbf{u}_{y} E_{0}\left\{\frac{1}{2 N+1} \exp \left(-j \beta_{1} \zeta\right)+\right. \\
\left.+\sum_{n=1}^{N} \frac{2}{2 N+1} \cos \left(n \Delta_{q} \xi\right) \exp \left(-j k_{n} \zeta\right)\right\}
\end{gathered}
$$

where $q_{n}=n \Delta_{q}, \Delta_{q}=2 \pi \xi_{\max }, k_{n}=\sqrt{\beta_{1}^{2}-n^{2} \Delta_{q}^{2}}$.

In fact the sum in (54) is the "periodic discrete numerical $\delta$-function"

$$
\varphi_{d}(\xi, 0)=\sin \left\{\left(N+\frac{1}{2}\right) \Delta_{q} \xi\right\} /(2 N+1) \sin \left(\frac{1}{2} \Delta_{q} \xi\right)
$$

with unit value in the center point $\xi=0$ and with zero values in the other $2 N$ "sampling points" $\xi_{k}=k \Delta_{\xi}$, $k= \pm 1, \pm 2, \ldots, \pm N$, where $\Delta_{\xi}=\xi_{\max } /(2 N+1)$ as depicted in Fig. 18 for $N=7$. 


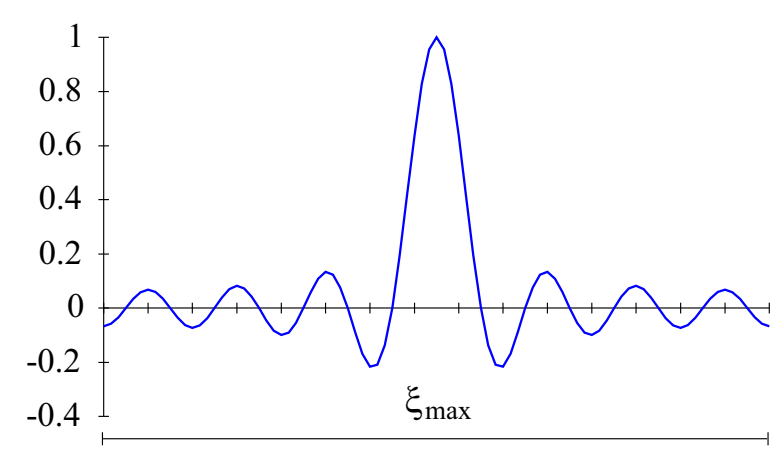

Fig. 18. The incident beam-wave-amplitude-pattern in the beam waist-plane $\varphi_{d}(\xi, 0)$ for $N=7$

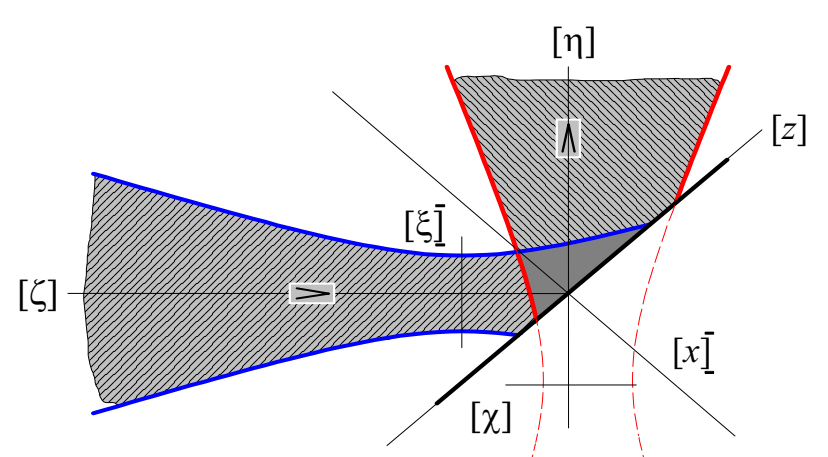

Fig. 19. Schematics of the beam total reflection with the GH-shift

Table 1. Angles (in degrees) $\Psi_{0 n}$, or $\Theta_{0 n}$ for TE configuration: A - reflected beam single boundary, B - shifted single boundary $\beta_{1} d_{2}=100^{\circ}, d_{1}=0, \mathrm{C}$ - frustrating slab $\beta_{1} d_{2}=100^{\circ}, d_{1} / d_{2}=$ $0.35, \mathrm{D}$ - detto $d_{1} / d_{2}=0.65, \mathrm{E} /$ detto $d_{1} / d_{2}=1$.

\begin{tabular}{cccccc}
$n$ & $\mathrm{~A}$ & $\mathrm{~B}$ & $\mathrm{C}$ & $\mathrm{D}$ & $\mathrm{E}$ \\
\hline 0 & 98.51 & -30.05 & 28.25 & 64.61 & 84.91 \\
1 & 98.49 & -29.55 & 21.81 & 54.87 & 78.49 \\
2 & 98.42 & -28.04 & 5.41 & 15.50 & 19.93 \\
3 & 98.28 & -25.50 & -11.93 & -27.38 & -51.00 \\
4 & 98.05 & -21.89 & -23.68 & -43.56 & -61.12 \\
5 & 97.64 & -17.16 & -30.08 & -49.09 & -63.26 \\
6 & 96.78 & -11.42 & -33.05 & -50.61 & -62.93 \\
7 & 93.52 & -6.32 & -34.85 & -50.67 & -61.48 \\
\hline
\end{tabular}

The reflected wave is expressed analogously as

$$
\begin{aligned}
& \boldsymbol{E}(\chi, \eta)=\mathbf{u}_{y} E_{0}(\chi, \eta)= \\
& =\mathbf{u}_{y} E_{0}\left\{\frac{1}{2 N+1} \exp \left\{-j\left(\beta_{1} \eta-\Psi_{00}\right)\right\}+\right. \\
& \left.+\sum_{n=1}^{N} \frac{2}{2 N+1} \cos \left(n \Delta_{q} \chi-\Psi_{n}\right) \exp \left\{-j\left(k_{n} \eta-\Psi_{0 n}\right)\right\}\right\},
\end{aligned}
$$

with the amplitude distribution in the reflected-beam waist plane

$$
\begin{aligned}
& \varphi_{r}(\chi, 0)=\frac{1}{2 N+1} \exp \left(j \Psi_{00}\right)+ \\
& +\sum_{n=1}^{N} \frac{2}{2 N+1} \cos \left(n \Delta_{q} \chi-\Psi_{n}\right) \exp \left(j \Psi_{0 n}\right) .
\end{aligned}
$$

or with $\Theta_{00}, \Theta_{01}, \ldots, \Theta_{0 N}$ and $\Theta_{1}, \Theta_{2}, \ldots, \Theta_{N}$ in case of frustrated reflection. The values of angles are shown in Tab. 1 and Tab. 2 for $\theta_{c}=10^{\circ}, \theta_{d 0}=50^{\circ}$ and $\Delta_{q} / \beta_{1}=0.09$.

Table 2. Angles $\Psi_{n}$, or $\Theta_{n}$ for the same parameters as in the Tab. 1

\begin{tabular}{cccccc}
$n$ & $\mathrm{~A}$ & $\mathrm{~B}$ & $\mathrm{C}$ & $\mathrm{D}$ & $\mathrm{E}$ \\
\hline 1 & 10.61 & 24.39 & 40.25 & 34.59 & 23.44 \\
2 & 21.32 & 48.89 & 82.16 & 91.93 & 95.61 \\
3 & 32.24 & 73.61 & 120.28 & 149.93 & 178.95 \\
4 & 43.54 & 98.70 & 150.24 & 179.99 & 201.11 \\
5 & 55.43 & 124.37 & 173.62 & 198.98 & 215.38 \\
6 & 68.36 & 151.09 & 193.26 & 214.14 & 227.60 \\
7 & 84.55 & 181.07 & 212.30 & 228.54 & 239.50 \\
\hline
\end{tabular}

The intensities of respective waves are shown in Fig. 20 and 21 . The intensity of single-boundary reflected-wave in Fig. 20 is due to GH-shift shifted with respect to the incident wave approximately one-quarter of the beam width, (if the beam width is defined as the distance between the first two central zero points), corresponding to the angle $\Psi_{7}=84.55^{\circ}$ in column A, row $n=7$, of Tab. 2 . One-quarter exactly would correspond to $\Psi_{7}=90^{\circ}$. The shifted-boundary reflected-wave is shifted approximately one-half of the beam width corresponding to the angle $\Theta_{7}=181.07^{\circ}$ for $\beta_{1} d_{2}=100^{\circ}$ (column B in Tab. 2).

The intensity patterns of the dielectric-slab-frustrated reflected waves in Fig. 21 clearly show that the originally single beam splits into a doublet corresponding to two total reflections, first on the boundary $1-2$ in Fig. 2 and the second on the boundary $3-4$. Notice that the magnitude of the reflection coefficient is, on the both boundaries, equal to one.

One part of the incident beam power is by a tunneling mechanism transferred by evanescent waves through the gap domain 2 to the dielectric slab and after being totally reflected on the boundary $3-4$ again by the tunneling mechanism returned into the domain 1 . Therefore obviously, the left peak corresponds to the reflection on the boundary $1-2$ and the right one to the boundary $3-4$. For $d_{1} / d_{2}=0.35$ (curve $c$ ) the reflection on the boundary $3-4$ dominates, the right peak is shifted farther to the right when compared with the case $c$ in Fig. 20 since the slab is shifted more to the right too.

When the gap is increased (curve $b, d_{1} / d_{2}=0.6$ ) the two reflection peaks are nearly equal and finally for 


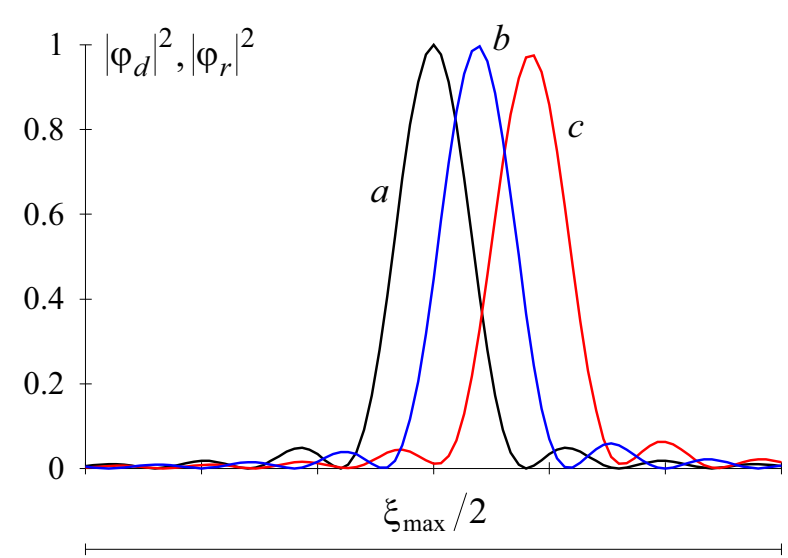

Fig. 20. Central portion(zoomed 2:1) of beam-intensity patterns at the beam waist of the (a) - incident wave, (b) - single-boundary reflected-wave, (c) - shifted-boundary reflected-wave $d_{1}=0$, $\beta_{1} d_{2}=100^{\circ}$, all in the TE configuration, for $\Delta_{q} / \beta_{1}=0.09, N=7$

curve $a, d_{1} / d_{2}=1$, the reflection on the boundary $3-4$ decreases, the power of reflected wave on the boundary 1-2 grows and gradually approaches the position of the peak $b$ in Fig. 20.

If the origin of the coordinate system of the incident beam $(\xi, \zeta)$ is placed in the point $\left(x_{0}, z_{0}\right)$, then the origin of the reflected beam coordinates $(\chi, \eta)$ is in the point $\left(-x_{0}, z_{0}\right)$, see Fig. 19. The planes $\zeta=0$ and $\eta=0$ are called "beam waists" and the amplitude distributions in the beam-waist's planes $\varphi_{d}(\xi, 0)(54)$ and $\varphi_{r}(\chi, 0)$ in $(58)$ determine fully the next course of propagating wave.

\section{Conclusions}

We have analysed and numerically modeled proximity effects when a dielectric slab is placed in the vicinity of a dielectric boundary, where total internal reflection occurs. It has been shown that the reflected beam-wave splits into a doublet due to two total reflections - on the original boundary and on the remote boundary of the slab. This leads in case of beam propagation to effectively wider beam and larger Goos-Hänchen shift.

\section{Acknowledgements}

This work has been supported by the Slovak Research and Development Agency under the contract no. APVV-

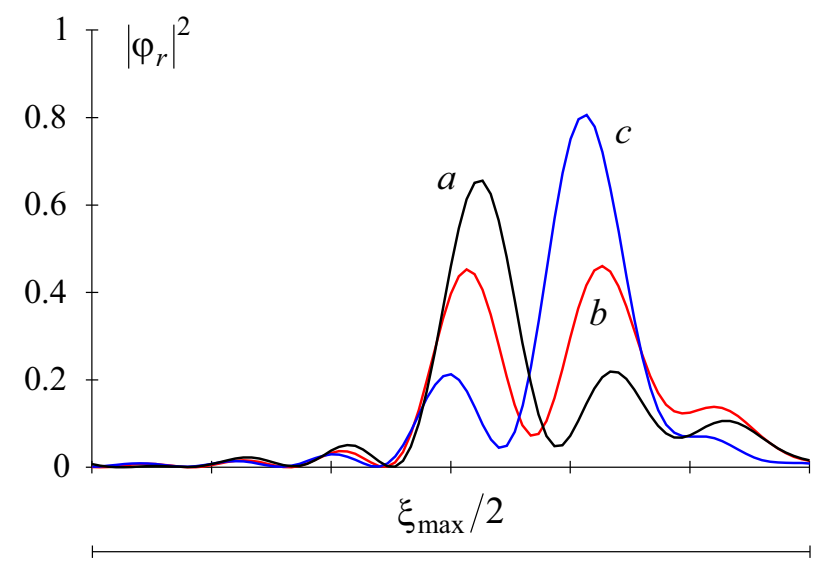

Fig. 21. Central portion(zoomed 2:1) of beam intensity patterns at the beam waist of the dielectric-slab-frustrated reflected-wave (a) $d_{1} / d_{2}=1,(\mathrm{~b})-d_{1} / d_{2}=0.65,(\mathrm{c})-d_{1} / d_{2}=0.35$, all in the TE configuration for $\beta_{1} d_{2}=100^{\circ}, \Delta_{q} / \beta_{1}=0.09, N=7$

16-0059 and by the Scientific Grant Agency of the Ministry of Education, Science, Research and Sport of the Slovak Republic and in part by the Slovak Academy of Sciences, under project no. 1/0405/16.

\section{REFERENCES}

[1] F. Goos and H. Hänchen, "Ein neuer und fundamentaler Versuch zur Totalreflexion, Ann, Phys, vol. 436, pp. 333-346 (1947).

[2] F. I. Fedorov, "K teorii polnogo otraženija , Dokl. Akad. Nauk SSSR vol. 105, pp. 465-468 (1955).

[3] B. R. Horowitz and T. Tamir, "Lateral Displacement of a Light Beam at a Dielectric Interface, J. Opt. Soc. Am. vol. 61, no. 5, pp. 586-594 (1971).

[4] A. W. Snyder and J. D. Love, "Goos-Hänchen shift, Appl. Opt. vol. 15 , no. 1 , pp. 236-238 (1976).

[5] V. G. Fedoseyev, "Energy motion on total internal reflection of an electromagnetic wave packet, J. Opt. Soc. Am. vol. A3, pp. 826-829 (1986).

[6] S. R. Seshadri, "Goos-Hänchen beam shift at total internal reflection, J. Opt. Soc. Am. vol. A5, no. 4, pp. 583-585 (1988).

[7] L'. Šumichrast, J. Franek, R. Dosoudil, and J. Červeňová, "Numerical modelling of the internal reflection and Goos-Hänchen shift of electromagnetic beam wave, JEE-EC vol. 69, no. 2, pp. 106-116 (2018).

Received 5 January 2019 